\title{
Shear-Induced Spin Polarization in Heavy-Ion Collisions
}

\author{
Baochi Fu๑,$^{1,2, \uparrow}$ Shuai Y. F. Liu $\odot,{ }^{3, *}$ Longgang Pang $\odot,{ }^{4, \$}$ Huichao Song $\odot,{ }^{1,2,5, \S}$ and Yi Yin $\oplus^{3,6, \|}$ \\ ${ }^{1}$ Department of Physics and State Key Laboratory of Nuclear Physics and Technology, Peking University, Beijing 100871, China \\ ${ }^{2}$ Collaborative Innovation Center of Quantum Matter, Beijing 100871, China \\ ${ }^{3}$ Quark Matter Research Center, Institute of Modern Physics, Chinese Academy of Sciences, Lanzhou 730000, China \\ ${ }^{4}$ Key Laboratory of Quark and Lepton Physics (MOE) and Institute of Particle Physics, Central China Normal University, \\ Wuhan 430079, China \\ ${ }^{5}$ Center for High Energy Physics, Peking University, Beijing 100871, China \\ ${ }^{6}$ University of Chinese Academy of Sciences, Beijing 100049, China
}

(Received 23 March 2021; revised 11 June 2021; accepted 16 August 2021; published 30 September 2021)

\begin{abstract}
We study the spin polarization generated by the hydrodynamic gradients. In addition to the widely studied thermal vorticity effects, we identify an undiscovered contribution from the fluid shear. This shearinduced polarization (SIP) can be viewed as the fluid analog of strain-induced polarization observed in elastic and nematic materials. We obtain the explicit expression for SIP using the quantum kinetic equation and linear response theory. Based on a realistic hydrodynamic model, we compute the differential spin polarization along both the beam direction $\hat{z}$ and the out-plane direction $\hat{y}$ in noncentral heavy-ion collisions at $\sqrt{s_{N N}}=200 \mathrm{GeV}$, including both SIP and thermal vorticity effects. We find that SIP contribution always shows the same azimuthal angle dependence as experimental data and competes with thermal vorticity effects. In the scenario that $\Lambda$ inherits and memorizes the spin polarization of a strange quark, SIP wins the competition, and the resulting azimuthal angle dependent spin polarization $P_{y}$ and $P_{z}$ agree qualitatively with the experimental data.
\end{abstract}

DOI: $10.1103 /$ PhysRevLett.127.142301

Introduction.-The transport phenomena involving spin are instrumental in investigating quantum effects in manybody systems. For example, the generation of spin current can be employed to probe intriguing properties of quantum materials [1]. In relativistic heavy-ion collisions, measuring spin polarization of hyperons has been proposed to explore the spin dynamics of quarks in the produced quark-gluon plasma (QGP) in heavy-ion collisions [2]. The observed $\Lambda$ spin polarization of the Relativistic Heavy Ion Collider (RHIC) and LHC experiments [3-7] opens a new avenue to study the hot and dense nuclear matter [8-36].

During the hydrodynamic evolution of the fireball created in heavy-ion collisions, the sizable gradients of hydrodynamic fields, such as temperature and flow gradient, could give rise to spin polarization. One widely studied effect is the spin polarization induced by thermal vorticity [37-39], a specific combination of temperature gradient and fluid vorticity. The large orbital angular momentum in noncentral heavy-ion collisions leads to nonzero vorticity and hence the global $\Lambda$ spin polarization [3,4]. The latter

Published by the American Physical Society under the terms of the Creative Commons Attribution 4.0 International license. Further distribution of this work must maintain attribution to the author(s) and the published article's title, journal citation, and DOI. Funded by SCOAP ${ }^{3}$. refers to the net polarization along the direction of the angular momentum of the fireball [i.e., the out-plane directions $(-\hat{y})]$ and has been successfully described by theories based on thermal vorticity effects. In addition, it has been proposed $[6,39,40]$ that the local structure of the vorticity and temperature gradient together with anisotropic flow can lead to local (differential) polarization along both the beam $(\hat{z})$ and the out-plane directions. Surprisingly, the predicted local $\Lambda$ spin polarization $[25,30,40]$ differs qualitatively from experimental observations [5,7]; see Refs. [6,24,27,41] for various attempts to resolve such "spin sign puzzle."

Nevertheless, vorticity and temperature gradient are not the only examples of hydrodynamic gradients. In this Letter, we identify the missing contribution to the spin polarization, namely, the effect of the shear stress tensor $\sigma^{\mu \nu}$. For certain elastic and nematic materials, the strain is expected to induce spin polarization, and such phenomenon has been observed; see Refs. [42-44] for examples. The shear-induced polarization (SIP) discussed in the present Letter may be viewed as an analog of such strain-induced polarization, but has never been discussed before. We will first show that SIP is allowed by symmetry and can also be derived explicitly based on quantum kinetic equation and the linear response theory. Then we employ the datacalibrated hydrodynamic model to investigate the phenomenological consequence of SIP, finding that SIP plays an 
essential role toward solving the spin sign puzzle in heavyion collisions.

Theory.-Following the formalism detailed in [36], we consider a system in the presence of slow varying flow velocity $u^{\mu}$ and temperature $T$ and look for gradient expansion of the axial Wigner function $\mathcal{A}^{\mu}$, which is related to the phase space density of spin polarization of fermions. Doing so allows us to study the response of spin polarization to hydrodynamic gradients.

We will first study the simplest case, that fermions are massless, using the expression from chiral kinetic theory [45-49]

$$
\mathcal{A}^{\mu}=\sum_{\lambda}\left(\lambda p^{\mu} f_{\lambda}+\frac{1}{2} \frac{\epsilon^{\mu \nu \alpha \rho} p_{\nu} u_{\alpha} \partial_{\rho} f_{\lambda}}{p \cdot u}\right),
$$

where $\lambda=+/-$ accounts for right- and left-handed chiral fermions, respectively, and where $p_{\mu}$ is the momentum and $f_{\lambda}$ is the distribution function of particles. The second term in Eq. (1) is commonly referred as the magnetization current term [50], since it can be written as $\nabla \times \boldsymbol{M}$ in medium rest frame where the $\boldsymbol{M}_{\lambda}=\int_{\boldsymbol{p}}(\hat{p} / 2|\boldsymbol{p}|) f_{\lambda}$ is the magnetic moments of chiral fermions. This magnetization current gives rise to various interesting transport phenomena, including the off-equilibrium chiral magnetic effect [51] and spin Hall effect [35,50,52], and has been recently implemented into a transport model for spin polarization [41].

Now, we replace $f_{\lambda}$ in Eq. (1) with local equilibrium distribution function $n\left(\beta\left(\varepsilon_{0}-\Delta \varepsilon_{\lambda}\right)\right)$, where the energy shift due to spin-vorticity coupling is given by $\Delta \varepsilon_{\lambda}=-(1 / 2) \lambda \omega \cdot p / \varepsilon_{0}$ with $\varepsilon_{0}=p \cdot u$, and $\omega^{\mu}=$ $(1 / 2) \epsilon^{\mu \nu \alpha \lambda} u_{\nu} \partial_{\alpha}^{\perp} u_{\lambda}$ is the vorticity. Here, $n(x)=1 /\left(e^{x}+1\right)$ denotes Fermi-Dirac function. Then, we can expand Eq. (1) to first order in gradient as

$$
\begin{aligned}
\mathcal{A}^{\mu}= & \beta n_{0}\left(1-n_{0}\right)\left(-\frac{1}{\varepsilon_{0}} p^{\mu} p^{\nu} \omega_{\nu}+\epsilon^{\mu \nu \alpha \rho} u_{\nu} p_{\alpha} \beta^{-1} \partial_{\rho} \beta\right. \\
& \left.-\frac{1}{\varepsilon_{0}} \epsilon^{\mu \nu \alpha \rho} u_{\nu} p_{\rho} p^{\lambda} \partial_{\alpha}^{\perp} u_{\lambda}\right),
\end{aligned}
$$

where $n_{0} \equiv n\left(\beta \varepsilon_{0}\right)$. Here, we denote a generic vector $V^{\mu}$ projected along the transverse direction with respect to $u^{\mu}$ as $V_{\perp}^{\mu}=\Delta^{\mu \nu} V_{\nu}$ where $\Delta^{\mu \nu}=\eta^{\mu \nu}-u^{\mu} u^{\nu}$ and $\eta^{\mu \nu}=$ $(1,-1,-1,-1)$ is the metric and $\epsilon^{0123}=1$. To proceed, we use $\partial_{\alpha}^{\perp} u_{\lambda}=\epsilon_{\alpha \lambda \gamma \zeta} u^{\gamma} \omega^{\zeta}+\partial_{(\alpha}^{\perp} u_{\lambda)}$ to evaluate the last term in Eq. (2), where $\partial_{(\alpha}^{\perp} u_{\lambda)} \equiv\left(\partial_{\alpha}^{\perp} u_{\lambda}+\partial_{\lambda}^{\perp} u_{\alpha}\right) / 2$. Noting $\left(-p_{\perp} \cdot \omega\right) u^{\mu}+\varepsilon_{0} \omega^{\mu}=(1 / 2) \epsilon^{\mu \nu \alpha \lambda} p_{\nu} \partial_{\alpha}^{\perp} u_{\lambda}$ and $p^{2}=0$ for massless fermions [53], we arrive at the desired expression

$$
\begin{aligned}
\mathcal{A}^{\mu}= & \frac{1}{2} \beta n_{0}\left(1-n_{0}\right)\left\{\epsilon^{\mu \nu \alpha \lambda} p_{\nu} \partial_{\alpha}^{\perp} u_{\lambda}\right. \\
& \left.+2 \epsilon^{\mu \nu \alpha \lambda} u_{\nu} p_{\alpha}\left[\beta^{-1}\left(\partial_{\lambda} \beta\right)\right]\right\}+\mathcal{A}_{\mathrm{SIP}}^{\mu},
\end{aligned}
$$

where the SIP as we advertised earlier is given by

$$
\begin{aligned}
\mathcal{A}_{\mathrm{SIP}}^{\mu} & =-\beta n_{0}\left(1-n_{0}\right) \frac{1}{\varepsilon_{0}} \epsilon^{\mu \nu \alpha \rho} u_{\nu} p_{\rho} p^{\lambda} \partial_{(\alpha}^{\perp} u_{\lambda)} \\
& =-\beta n_{0}\left(1-n_{0}\right) \frac{p_{\perp}^{2}}{\varepsilon_{0}} \epsilon^{\mu \nu \alpha \rho} u_{\nu} Q_{\alpha}^{\lambda} \sigma_{\rho \lambda} .
\end{aligned}
$$

From the first line to the second line in Eq. (4), we have used $p^{\mu}=\varepsilon_{0} u^{\mu}+p_{\perp}^{\mu}$. The generalized quadrupole tensor and shear stress tensor are given by $Q^{\mu \nu} \equiv-p_{\perp}^{\mu} p_{\perp}^{\nu} / p_{\perp}^{2}+\Delta^{\mu \nu} / 3$ and $\sigma^{\mu \nu}=\partial_{\perp}^{(\mu} u^{\nu)}-\Delta^{\mu \nu} \partial \cdot u / 3$, respectively. Note that $\mathcal{A}_{\mathrm{SIP}}^{\mu}$ solely arises from the magnetization current term.

To extend our analysis to fermions with an arbitrary mass, we consider the linear response theory (detailed in Ref. [36]) and find that Eq. (3) applies equally to massless and massive fermions. This means that the axial Wigner function $\mathcal{A}^{\mu}$ only inexplicitly depends on the fermion mass through the mass dependence of $p^{\mu}$. The key in the linear response analysis is to match the gradient expansion of $\mathcal{A}^{\mu}$ to small frequency and wave vector behavior of the retarded correlator $G_{R}^{\alpha ; \mu \nu}=$ $\left\langle\left[\bar{\psi}(\boldsymbol{x}-\boldsymbol{y} / 2) \gamma^{\alpha} \gamma^{5} \psi(\boldsymbol{x}+\boldsymbol{y} / 2), \hat{T}^{\mu \nu}(0, \mathbf{0})\right]\right\rangle \theta(t)$, where $\hat{T}^{\mu \nu}$ denotes the stress-energy tensor and $\psi$ is the fermionic field. Using one loop calculation of $G_{R}^{\alpha ; \mu \nu}$, we confirm Eq. (3) for fermions with arbitrary mass.

For the convenience of the subsequent discussion, we combine the first two terms in " \{\} " in Eq. (3) using the hydrodynamic equation $(u \cdot \partial) u_{\alpha}=-\beta^{-1} \partial_{\alpha}^{\perp} \beta+\mathcal{O}\left(\partial^{2}\right)$ to obtain

$$
\mathcal{A}^{\mu}=\frac{1}{2} n_{0}\left(1-n_{0}\right) \epsilon^{\mu \nu \alpha \lambda} p_{\nu} \partial_{\alpha}\left(\beta u_{\lambda}\right)+A_{\mathrm{SIP}}^{\mu},
$$

where the first term reproduces the spin polarization induced by thermal vorticity, as was studied by many authors [37-39]. Our new finding is the second term, which describes the effects of the shear stress tensor on spin polarization.

To illustrate the qualitative feature of SIP, let us consider a standard shear flow profile $\partial_{x} u_{y} \neq 0$ (in fluid rest frame). Then, SIP predicts a quadrupole pattern in the local (differential) spin polarization along the $z$ direction, i.e., $\mathcal{P}_{z} \propto\left(p_{x}^{2}-p_{y}^{2}\right) \partial_{x} u_{y}$. This simple example clearly demonstrates that SIP plays an essential role on local (differential) spin polarization.

Two scenarios.-To quantitatively predict the $\Lambda$ spin polarization due to hydrodynamic gradient effects, we need to hadronize the polarized strange quarks into Lambda hyperons followed by hadronic evolution toward the kinetic freeze-out. However, the development of hadronization and transport models that consistently include the spin degrees of freedom is highly nontrivial; see Refs. [21,22] for recent progresses. In order not to introduce complicated model assumptions, we shall consider two widely assumed 
limiting scenarios $[2,25,28,29,39,40,54]$ and focus not on the magnitude but on the qualitative features of the resulting azimuthal angle dependence of spin polarization.

In the first scenario, namely the "Lambda equilibrium," we assume the spin relaxation rate is large enough so that $\Lambda$ hyperons immediately respond to the presence of hydrodynamic gradients once $\Lambda$ are formed through hadronization $[25,39,40,54]$.

Turning to the second scenario, we consider the opposite limit that $\Lambda$ "inherits" the spin polarization from its constituent strange quark [2,55-57], and the resulting spin polarization is frozen ever since the hadronization. This scenario will be referred to as the "strange memory." Following Refs. [2,55], we shall use the relation $P_{\Lambda}=P_{s}$ based on a quark-recombination model to evaluate $\Lambda$ polarization. See Refs. $[28,29,41]$ for other studies based on this strange memory picture. In reality, $\Lambda$ spin polarization should evolve from the strange memory scenario toward the Lambda equilibrium scenario. Therefore, comparing results from those two benchmark scenarios provides us qualitative guidance on what we anticipate to observe in heavy-ion collisions.

Guided by Refs. [37,38], we shall use the following freeze-out prescription to connect the axial Wigner function given by Eq. (3) to spin polarization vector $P^{\mu}$ on the freeze-out hypersurface $\Sigma_{\mu}$ :

$$
P^{\mu}(\boldsymbol{p})=\frac{\int d \Sigma^{\alpha} p_{\alpha} \mathcal{A}^{\mu}(x, \boldsymbol{p} ; m)}{2 m \int d \Sigma^{\alpha} p_{\alpha} n\left(\beta \varepsilon_{0}\right)}
$$

Here the factor of 2 in the denominator counts two states of the spin- $1 / 2$ fermions. In the Lambda equilibrium (strange memory) scenario, we shall compute $\Lambda$ (strange quark) spin polarization using Eq. (6). We use $m=$ $1.116 \mathrm{GeV}$ for Lambda mass and the benchmark value for the strange quark mass $m=0.3 \mathrm{GeV}$, respectively; the latter is between strange current and constituent mass (cf. Refs. [58-62]). In principle, we should compute Eq. (6) at the kinetic freeze-out for the Lambda equilibrium scenario, but we have checked that the results of doing so are qualitatively similar to those calculated at chemical freeze-out. To simplify the comparison, we shall show the spin polarization vector computed at chemical freeze-out in both scenarios.

Results. - In this Letter, we implement $3+1-d$ hydrodynamics MUSIC [63-65] with AMPT initial conditions [66-68] to generate the freeze-out surface and associated temperature and flow velocity profiles for the spin polarization calculation described by Eq. (3). Unless noted otherwise, we use the same inputs and parameter sets as used in a previous paper [25], where the Lambda equilibrium scenario without SIP has been studied. Such hydrodynamic calculations have been well calibrated to fit the $d N_{\mathrm{ch}} / d y, p_{T}$ spectra and $v_{2}\left(p_{T}\right)$ of pions and protons in $\mathrm{Au}-\mathrm{Au}$ collisions at $\sqrt{s_{N N}}=200 \mathrm{GeV}$. [69]. Below, we will use this hydrodynamic framework to calculate the azimuthal angle $\phi_{p}$ dependence spin of polarization vector along the beam direction $P_{z}\left(\phi_{p}\right)$ and along the out-plane direction $P_{y}\left(\phi_{p}\right)$ with centrality $20 \%-60 \%$ [5] and $20 \%-$ $50 \%$ [7], respectively.

In Figs. 1 and 2, we plot the local spin polarization vector $P_{z}\left(\phi_{p}\right)$ and $P_{y}\left(\phi_{p}\right)$ in the particle rest frame. They include the separate and/or combined effects from SIP, fluid vorticity, and temperature gradient. Although the thermal vorticity contribution has been studied extensively, this is the first time that the signature of SIP has been investigated in heavy-ion collisions. All curves contributing to $P_{z}$ and $\left(-P_{y}\right)$ can be parametrized approximately as " $b_{z} \sin \left(2 \phi_{p}\right)$ " and " $a+b_{y} \cos \left(2 \phi_{p}\right)$," respectively. For the transparency of comparing with the qualitative features of the experimental results shown in Fig. 2, we shall refer to a contribution as the "same sign" ("opposite sign") when $b_{z}, b_{y}>0\left(b_{z}, b_{y}<0\right)$.
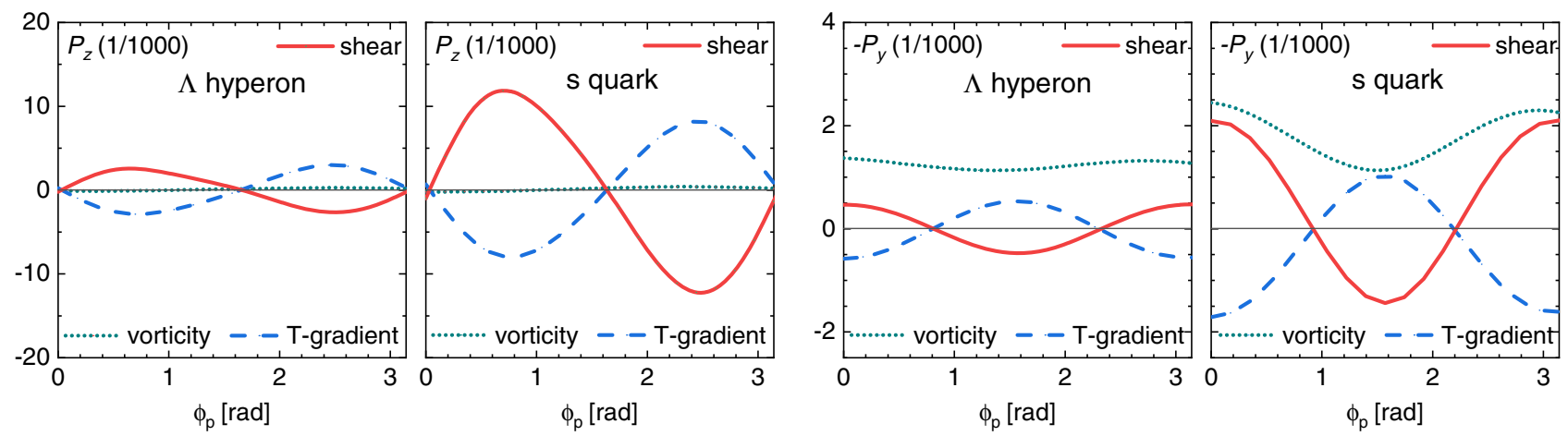

FIG. 1. Spin polarization as a function of azimuthal angle $\phi_{p}$ along $z$ and $y$ directions, induced by hydrodynamic gradients for $\Lambda$ hyperon and strange quarks at the freeze-out surface: colored curves show effects from vorticity, temperature gradient, and shear stress tensor (i.e., the SIP), corresponding to the first, second, and third terms in Eq. (3), respectively. The effects of thermal vorticity are given by the sum of vorticity and temperature gradient effects and have been studied by many others. SIP is the new effect studied in this Letter and competes with the thermal vorticity effects. 



FIG. 2. Spin polarization of $\Lambda$ hyperon (left) and strange quark (middle) along $z$ (upper) and $y$ (lower) directions induced by the combined effects of the shear stress tensor and thermal vorticity (solid curves) and by thermal vorticity effects only (dashed curves) on the freeze-out surface. Right: the replotted experimental data in Refs. [5,7]. The $P_{z}$ is converted using $P_{z}=\left\langle\cos \theta_{p}^{*}\right\rangle /\left[\alpha_{H}\left\langle\left(\cos \theta_{p}^{*}\right)^{2}\right\rangle\right]$ assuming zero error in the denominator. The results for strange spin polarization illustrate the anticipated qualitative behavior in the strange memory scenario, i.e.; the memory of strange quark polarization is preserved in the measured $\Lambda$ polarization.

In Fig. 1, we plot spin polarization induced by the shear stress tensor (SIP), vorticity (VoIP), and temperature gradient (TIP) [cf. Eq. (3)] in both Lambda equilibrium and strange memory scenarios. We observe that the VoIP gives the dominant contribution to global polarization and those from SIP and TIP are largely canceled with each other. However, the azimuthal angle dependence of $P_{z}$ and $P_{y}$ arises mostly from SIP and TIP. As a marked qualitative feature, SIP always leads to the same sign contribution to spin polarization in both $z$ and $y$ directions. On the other hand, the effects of thermal vorticity on the azimuthal angle dependence of spin polarization are dominated by TIP, which shows the opposite sign behavior. That thermal vorticity leads to the opposite sign, as seen in many early studies $[25,30,40]$, is sometimes referred to as the spin sign puzzle.

Given that SIP contribution is comparable to TIP contribution in magnitude but is qualitatively different in sign, the competition between SIP and TIP will eventually determine the azimuthal angle dependence of the total spin polarization. This competition is best seen in Fig. 2, where the total spin polarization as a function of $\phi_{p}$ is drastically different with and without SIP. In the Lambda equilibrium scenario, though, TIP wins the competition and the total polarization is the opposite sign, even in the presence of SIP. However, SIP becomes more important when the mass of the spin carrier becomes smaller. This can be easily understood from Eqs. (4) and (5) that the factor $\left|p_{\perp}^{2}\right| / \varepsilon_{0}^{2}$ (the square of typical velocity of fermions) is larger when the spin carrier is lighter. Indeed, in the strange memory scenario, SIP prevails over TIP in both $P_{z}$ and $P_{y}$. Seeing this, we should not be surprised to find that the total spin polarization shows the same sign.

To complement Figs. 1 and 2, we compare the spin polarization vector in the particle rest frame $\boldsymbol{P}_{\text {rest }}$ as shown above with those in the lab frame $\boldsymbol{P}_{\text {lab }}$ in Fig. 3, where we have used the relation $\boldsymbol{P}_{\text {rest }}=\boldsymbol{P}_{\text {lab }}-$ $\{\boldsymbol{p} /[\varepsilon(\varepsilon+m)]\}\left(\boldsymbol{P}_{\text {rest }} \cdot \boldsymbol{p}\right)$. This comparison is of particular relevance for the strange memory scenario because the

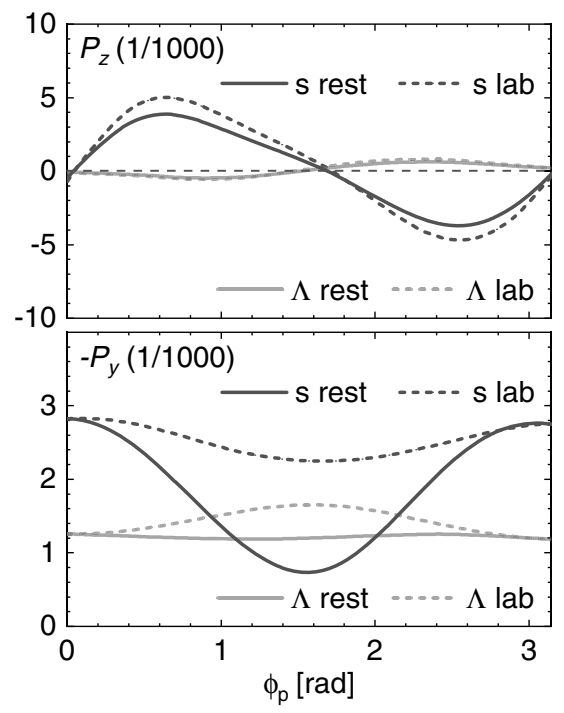

FIG. 3. The comparison between strange quark and $\Lambda$ hyperon spin polarization in lab and particle rest frame. 
relation $P_{\Lambda}=P_{s}$ we use in this scenario has an ambiguity on which frame it applies to. The future development on the hadronization of spin should remove such ambiguity. We estimate uncertainty from this ambiguity by examining spin polarization in both the particle rest frame and the lab frame. We notice that quantitatively, $P_{z}$ is insensitive to the frame choice, while the influence of this choice on $P_{y}$ is more significant. For discussion on other subtle points associated with the frame choice, see Refs. [41,70].

To investigate the generality of the results reported above, we repeat our calculations by systematically varying inputs for the present hydrodynamic model, including initial conditions, freeze-out temperature, and the parametrization of the shear viscosity, bulk viscosity, and equation of state (EoS); see our upcoming publication for more details [71]. In all cases that we have explored, SIP always induces the same sign contribution, whereas the TIP contribution is the opposite sign. Moreover, in the Lambda equilibrium scenario, the total spin polarization is the opposite sign. This should be contrasted with the results seen in the strange memory scenario. Except for some specific cases when a unusually large $T$ gradient is caused by EoSs much harder than the lattice EoS or a sharply peaked bulk viscosity around the freeze-out region, the azimuthal angle dependence of spin polarization is generically dominated by SIP and shows the qualitative agreement with the data.

Summary and discussion. - To summarize, we discover a new mechanism to generate spin polarization in a fluid, namely, shear-induced polarization (SIP). For the first time, we investigate the phenomenological implication of SIP. We find that the SIP consistently gives rise to the same sign as the differential Lambda polarization observed in the experiment and is comparable to thermal vorticity effects in magnitude. By comparing the results from two commonly used scenarios, i.e., the Lambda equilibrium and strange memory scenarios, we observe that the former fails to describe the data. In contrast, the strange memory scenario results qualitatively agree with data. Note that, without SIP, none of the scenarios describe the data. Therefore, it is tempting to conclude that SIP is essential to resolve the spin sign puzzle in heavy-ion collisions. The qualitative agreement of the strange memory scenario results with data should point to a number of interesting future directions.

For future quantitative studies, one should establish a microscopic dynamical framework that can convert polarized quarks to a polarized $\Lambda$ and incorporate the subsequent evolution in the hadronic stage [15-22]. In addition, the nonperturbative effects on spin polarization should also be explored. Future studies should also investigate the evolution of quark spin polarization in QGP [10-14]. We limit our calculations to high-energy, heavy-ion collisions. SIP should be present in collisions at the beam scan energies at RHIC and could potentially be employed to explore the properties of QCD matter at finite baryon density.
We are thankful for helpful discussions with Shanshan Cao, Xu Cao, Hengtong Ding, Fei Gao, Feng Li, Yanfang Liu, Subahash Singha, and Yifeng Sun. This work was supported in part by the NSFC under Grants No. 12075007 and No. 11675004 (B.F. and H.S.) and by No. 11861131009 and No. 12075098 (L. P.), as well as by the Strategic Priority Research Program of Chinese Academy of Sciences, Grant No. XDB34000000 (S. Y.F.L. and Y. Y.). We acknowledge the extensive computing resources provided by the Supercomputing Center of Chinese Academy of Science (SCCAS), Tianhe-1A from the National Supercomputing Center in Tianjin, China, and the High-Performance Computing Platform of Peking University, as well as Nuclear Science Computer Center at CCNU (NSC3), China.

* Corresponding author.

lshphy@gmail.com

fubaochi@pku.edu.cn

trpang@mail.ccnu.edu.cn

\$huichaosong@pku.edu.cn

"yiyin@impcas.ac.cn

[1] W. Han, S. Maekawa, and X.-C. Xie, Nat. Mater. 19, 139 (2020).

[2] Z.-T. Liang and X.-N. Wang, Phys. Rev. Lett. 94, 102301 (2005); 96, 039901(E) (2006).

[3] L. Adamczyk et al. (STAR Collaboration), Nature (London) 548, 62 (2017).

[4] J. Adam et al. (STAR Collaboration), Phys. Rev. C 98, 014910 (2018).

[5] J. Adam et al. (STAR Collaboration), Phys. Rev. Lett. 123, 132301 (2019).

[6] S. A. Voloshin, EPJ Web Conf. 171, 07002 (2018).

[7] T. Niida (STAR Collaboration), Nucl. Phys. A982, 511 (2019).

[8] F. Becattini and M. A. Lisa, Annu. Rev. Nucl. Part. Sci. 70, 395 (2020).

[9] X.-G. Huang, J. Liao, Q. Wang, and X.-L. Xia, Lect. Notes Phys. 987, 281 (2021).

[10] W. Florkowski, B. Friman, A. Jaiswal, and E. Speranza, Phys. Rev. C 97, 041901(R) (2018).

[11] K. Hattori, M. Hongo, X.-G. Huang, M. Matsuo, and H. Taya, Phys. Lett. B 795, 100 (2019).

[12] K. Fukushima and S. Pu, Phys. Lett. B 817, 136346 (2021).

[13] S. Li, M. A. Stephanov, and H.-U. Yee, Phys. Rev. Lett. 127, 082302 (2021).

[14] S. Bhadury, J. Bhatt, A. Jaiswal, and A. Kumar, Eur. Phys. J. Spec. Top. 230, 655 (2021).

[15] S. Li and H.-U. Yee, Phys. Rev. D 100, 056022 (2019).

[16] Z. Wang, X. Guo, and P. Zhuang, arXiv:2009.10930.

[17] D.-L. Yang, K. Hattori, and Y. Hidaka, J. High Energy Phys. 07 (2020) 070.

[18] N. Weickgenannt, E. Speranza, X.-1. Sheng, Q. Wang, and D. H. Rischke, Phys. Rev. Lett. 127, 052301 (2021).

[19] N. Weickgenannt, E. Speranza, X.-l. Sheng, Q. Wang, and D. H. Rischke, Phys. Rev. D 104, 016022 (2021). 
[20] F. Li and S. Y. Liu, arXiv:2004.08910.

[21] X.-L. Sheng, Q. Wang, and X.-N. Wang, Phys. Rev. D 102, 056013 (2020).

[22] Z. Wang and P. Zhuang, arXiv:2101.00586.

[23] R. Singh, G. Sophys, and R. Ryblewski, Phys. Rev. D 103, 074024 (2021).

[24] H.-Z. Wu, L.-G. Pang, X.-G. Huang, and Q. Wang, Phys. Rev. Research 1, 033058 (2019).

[25] B. Fu, K. Xu, X.-G. Huang, and H. Song, Phys. Rev. C 103, 024903 (2021).

[26] W. Florkowski, A. Kumar, R. Ryblewski, and R. Singh, Phys. Rev. C 99, 044910 (2019).

[27] W. Florkowski, A. Kumar, A. Mazeliauskas, and R. Ryblewski, Phys. Rev. C 100, 054907 (2019).

[28] Y. Sun and C. M. Ko, Phys. Rev. C 96, 024906 (2017).

[29] Y. Sun and C. M. Ko, Phys. Rev. C 99, 011903(R) (2019).

[30] X.-L. Xia, H. Li, Z.-B. Tang, and Q. Wang, Phys. Rev. C 98, 024905 (2018).

[31] J.-j. Zhang, R.-h. Fang, Q. Wang, and X.-N. Wang, Phys. Rev. C 100, 064904 (2019).

[32] E. Speranza and N. Weickgenannt, Eur. Phys. J. A 57, 155 (2021).

[33] L. P. Csernai, J. I. Kapusta, and T. Welle, Phys. Rev. C 99, 021901(R) (2019).

[34] F. Becattini, in Strongly Interacting Matter under Rotation, edited by F. Becattini, J. Liao, and M. Lisa, Lecture Notes in Physics Vol. 987 (Springer, Cham, 2021), pp. 15-52, https:// doi.org/10.1007/978-3-030-71427-7_2.

[35] S. Y. F. Liu and Y. Yin, arXiv:2006.12421.

[36] S. Y. F. Liu and Y. Yin, J. High Energy Phys. 07 (2021) 188.

[37] F. Becattini, V. Chandra, L. Del Zanna, and E. Grossi, Ann. Phys. (Amsterdam) 338, 32 (2013).

[38] R.-h. Fang, L.-g. Pang, Q. Wang, and X.-n. Wang, Phys. Rev. C 94, 024904 (2016).

[39] L.-G. Pang, H. Petersen, Q. Wang, and X.-N. Wang, Phys. Rev. Lett. 117, 192301 (2016).

[40] F. Becattini and I. Karpenko, Phys. Rev. Lett. 120, 012302 (2018).

[41] S. Y. F. Liu, Y. Sun, and C. M. Ko, Phys. Rev. Lett. 125, 062301 (2020).

[42] S. A. Crooker and D. L. Smith, Phys. Rev. Lett. 94, 236601 (2005).

[43] A. G. Mal'shukov, C. S. Tang, C. S. Chu, and K. A. Chao, Phys. Rev. Lett. 95, 107203 (2005).

[44] T. Kissikov, R. Sarkar, M. Lawson, B. T. Bush, E. I. Timmons, M. A. Tanatar, R. Prozorov, S. L. Bud'ko, P. C. Canfield, R. M. Fernandes, and N. J. Curro, Nat. Commun. 9, 1058 (2018).

[45] D. T. Son and N. Yamamoto, Phys. Rev. Lett. 109, 181602 (2012).

[46] M. A. Stephanov and Y. Yin, Phys. Rev. Lett. 109, 162001 (2012).
[47] J.-Y. Chen, D. T. Son, M. A. Stephanov, H.-U. Yee, and Y. Yin, Phys. Rev. Lett. 113, 182302 (2014).

[48] J.-Y. Chen, D. T. Son, and M. A. Stephanov, Phys. Rev. Lett. 115, 021601 (2015).

[49] Y. Hidaka, S. Pu, and D.-L. Yang, Phys. Rev. D 97, 016004 (2018).

[50] K. Hattori, Y. Hidaka, and D.-L. Yang, Phys. Rev. D 100, 096011 (2019).

[51] D. E. Kharzeev, M. A. Stephanov, and H.-U. Yee, Phys. Rev. D 95, 051901(R) (2017).

[52] D. T. Son and N. Yamamoto, Phys. Rev. D 87, 085016 (2013).

[53] We have used $p^{2}=0$ to make the connection between the results from massless case and that of massive theory more transparent.

[54] I. Karpenko and F. Becattini, Eur. Phys. J. C 77, 213 (2017).

[55] J.-H. Gao, Z.-T. Liang, Q. Wang, and X.-N. Wang, in Strongly Interacting Matter under Rotation, edited by $\mathrm{F}$. Becattini, J. Liao, and M. Lisa, Lecture Notes in Physics Vol. 987 (Springer, Cham, 2021), pp. 194-246, https://doi .org/10.1007/978-3-030-71427-7_7.

[56] B. K. Jennings, Phys. Lett. B 246, 325 (1990).

[57] J. Cohen and H. J. Weber, Phys. Rev. C 44, 1181 (1991).

[58] W.-j. Fu, J. M. Pawlowski, and F. Rennecke, Phys. Rev. D 101, 054032 (2020).

[59] J. D. Carroll, D. B. Leinweber, A. G. Williams, and A. W. Thomas, Phys. Rev. C 79, 045810 (2009).

[60] F. Gao and Y.-x. Liu, Phys. Rev. D 97, 056011 (2018).

[61] X. Li, W.-J. Fu, and Y.-X. Liu, Phys. Rev. D 99, 074029 (2019).

[62] W. Zhao, C. M. Ko, Y.-X. Liu, G.-Y. Qin, and H. Song, Phys. Rev. Lett. 125, 072301 (2020).

[63] B. Schenke, S. Jeon, and C. Gale, Phys. Rev. C 82, 014903 (2010).

[64] B. Schenke, S. Jeon, and C. Gale, Phys. Rev. Lett. 106, 042301 (2011).

[65] B. Schenke, S. Jeon, and C. Gale, Phys. Rev. C 85, 024901 (2012).

[66] Z.-W. Lin, C. M. Ko, B.-A. Li, B. Zhang, and S. Pal, Phys. Rev. C 72, 064901 (2005).

[67] L. Pang, Q. Wang, and X.-N. Wang, Phys. Rev. C 86, 024911 (2012).

[68] H.-j. Xu, Z. Li, and H. Song, Phys. Rev. C 93, 064905 (2016).

[69] We have also performed an independent calculation using CLVisc [39] hydrodynamics framework and produced all the key features shown in the main text.

[70] W. Florkowski and R. Ryblewski, arXiv:2102.02890.

[71] B. Fu, S. Y. F. Liu, L. Pang, H. Song, and Y. Yin (to be published). 